\title{
Supporting Information: Hydrogen sensing using thin-film perfect light absorber
}

\author{
Mohamed ElKabbash ${ }^{1,2^{*}}$, Kandammathe Valiyaveedu Sreekanth ${ }^{1}$, Yunus Alapan ${ }^{3}$, Myeongseop Kim ${ }^{3}$, \\ Jonathan $\mathrm{Cole}^{4}$, Arwa Fraiwan ${ }^{3}$, Theodore Letsou ${ }^{1}$, Yandong Li ${ }^{1}$, Chunlei Guo ${ }^{2,5}$ R. Mohan Sankaran ${ }^{4}$, \\ Umut A. Gurkan $3,6,7,8$, Michael Hinczewski ${ }^{1}$, Giuseppe Strangi ${ }^{1,9,10^{*}}$.
}

1. Department of Physics, Case Western Reserve University, 10600 Euclid Avenue, Cleveland, Ohio 44106, USA.

2. The Institute of Optics, University of Rochester, Rochester, NY 14627, USA.

3. Case Biomanufacturing and Microfabrication Laboratory, Mechanical and Aerospace Engineering Department, Case Western Reserve University, Cleveland, Ohio 44106.

4. Department of Chemical and Biomolecular Engineering, Case Western Reserve University, Cleveland, OH, USA.

5. GPL, State Key Laboratory of Applied Optics, Changchun Institute of Optics, Fine Mechanics and Physics, Chinese Academy of Sciences, Changchun 130033, China.

6. Biomedical Engineering Department, Case Western Reserve University, Cleveland, Ohio 44106, USA.

7. Department of Orthopedics, Case Western Reserve University, Cleveland, Ohio 44106, USA.

8. Advanced Platform Technology Center, Louis Stokes Cleveland Veterans Affairs Medical Center, Cleveland, Ohio 44106, USA.

9. CNR-NANOTEC, Istituto di Nanotecnologia and Department of Physics, University of Calabria, Italy.

10. Istituto Italiano di Tecnologia, Via Morego 30, 16163, Genova, Italy.

*Correspondence authors E-mail: mke23@,case.edu, gxs284@,case.edu. 


\section{Sensor's performance at short-wave infrared wavelengths:}

As we discussed in the manuscript, the gas sensor's sensitivity relies on the change in the complex refractive index of $\mathrm{Pd}$ when it forms a hydride. Figure $2 \mathrm{c}$ in the manuscript shows that the change in refractive index is larger for longer wavelengths. Consequently, a sensor operating at longer wavelengths is expected to have higher sensitivity. We demonstrate this by fabricating two cavities realizing perfect light absorption at $\sim 1700 \mathrm{~nm}$ and $2100 \mathrm{~nm}$ as shown in Fig. S1a, and Fig. S1b, respectively. Compared to the sensor presented in the manuscript which showed $\sim 60 \mathrm{~nm}$ shift in $\lambda_{\min }$ for $\mathrm{H}_{2}(4 \%)$, these sensors exhibit $88 \mathrm{~nm}$ and $170 \mathrm{~nm}$ shift, respectively.

a

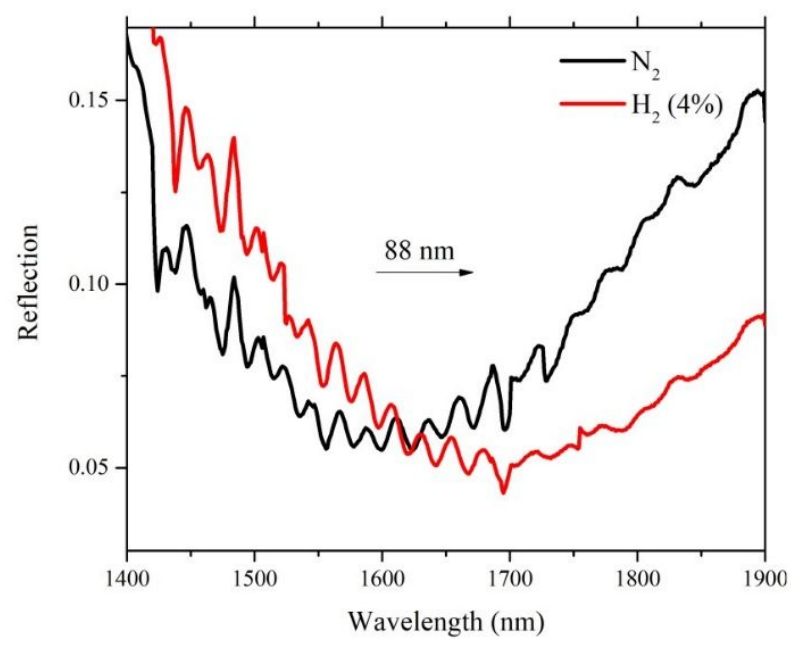

b

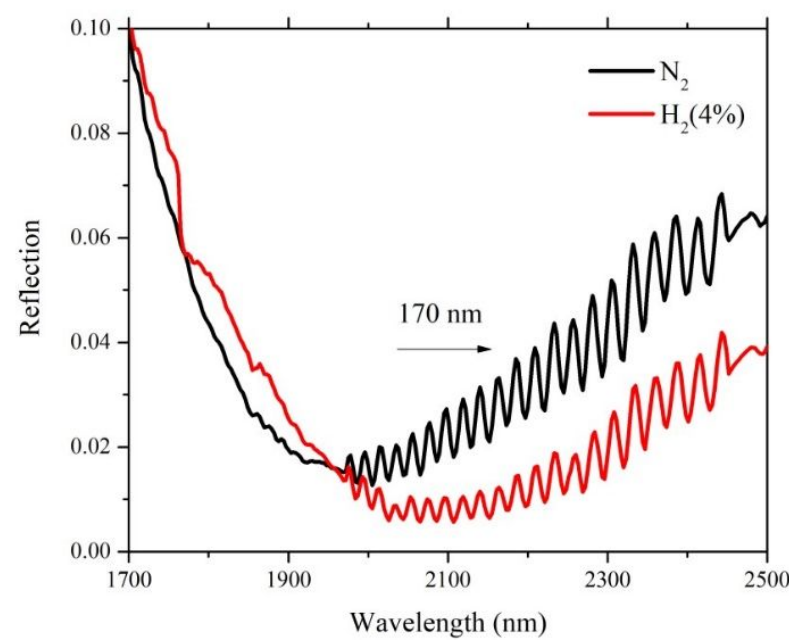

Figure S1: Measured reflection spectrum for an MDM cavity with $\mathrm{N}_{2}$ (black line) and with $\mathrm{H}_{2}(4 \%)$ (red line) with perfect absorption occurring at (a) $1700 \mathrm{~nm}$, and (b) $2100 \mathrm{~nm}$.

We note that the measured reflection in Fig. S1 shows modulation in intensity. This problem is also present when operating using a similar sample to that presented in the manuscript as shown in Fig. S2a. The observed modulation is due to interference effect from light reflected from the channel and from the sample similar to what one obtains from a shearing interferometer 
using a microfluidic channel with thickness $\sim 50 \mathrm{~mm}$ (see Fig. R2b). To attenuate this effect and obtain the results shown in the paper, we used a thicker channel with height $\sim 100 \mathrm{~mm}$. Using thicker microfluidic channel enabled the separation between the spot reflected from the channel and from the sensor surface.

a
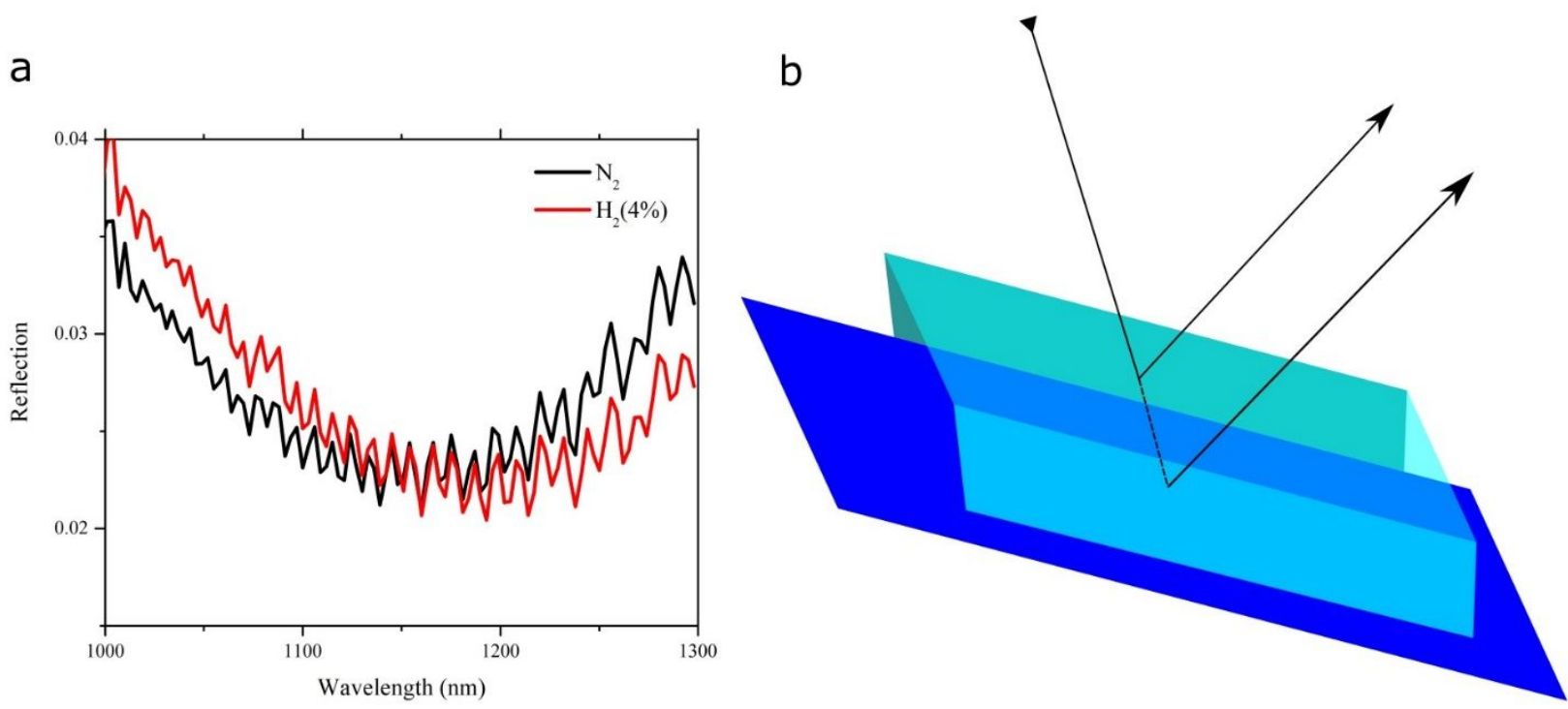

Figure S2: (a) Measured reflection of an MDM cavity with only $\mathrm{N}_{2}$ (black line) and after introducing $\mathrm{H}_{2}(4 \%)$, using a microfluidic channel with height $\sim 50 \mu \mathrm{m}$. The observed periodic modulation in the measured reflection is due to interference from the light reflected off the channel and the sample as shown in (b).

\section{Determining the perfect light absorption angle:}

In the cavity demonstrated in the manuscript, light undergoes near perfect light absorption across a wide range of wavelengths and angles. For optimal results, we measured the angular reflection from $15^{\circ}$ to $55^{\circ}$ with $5^{\circ}$ angular resolution to determine the optimal angle to operate our sensor as shown in Fig. S3a (and Fig. 2c in the manuscript). Then we measured the angular reflection with higher angular resolution of $1^{\circ}$ to obtain the optimal angle as shown in Fig. S3b. 
a

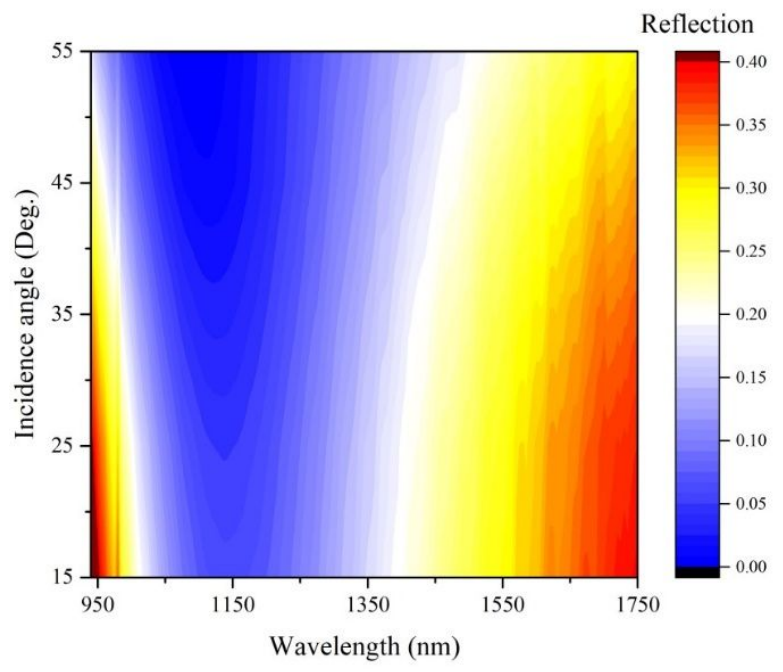

b

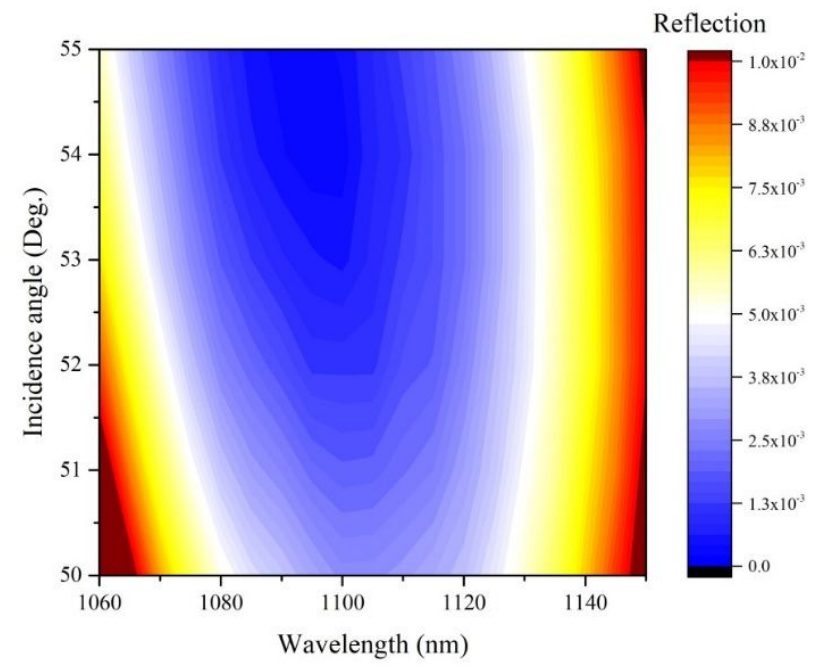

Figure S3: Angular reflection measurement for the cavity gas sensor without channel measured from (a) $15^{\circ}$ to $55^{\circ}$ with $5^{\circ}$ angular resolution, and (b) from $50^{\circ}$ to $55^{\circ}$ with $1^{\circ}$ angular resolution.

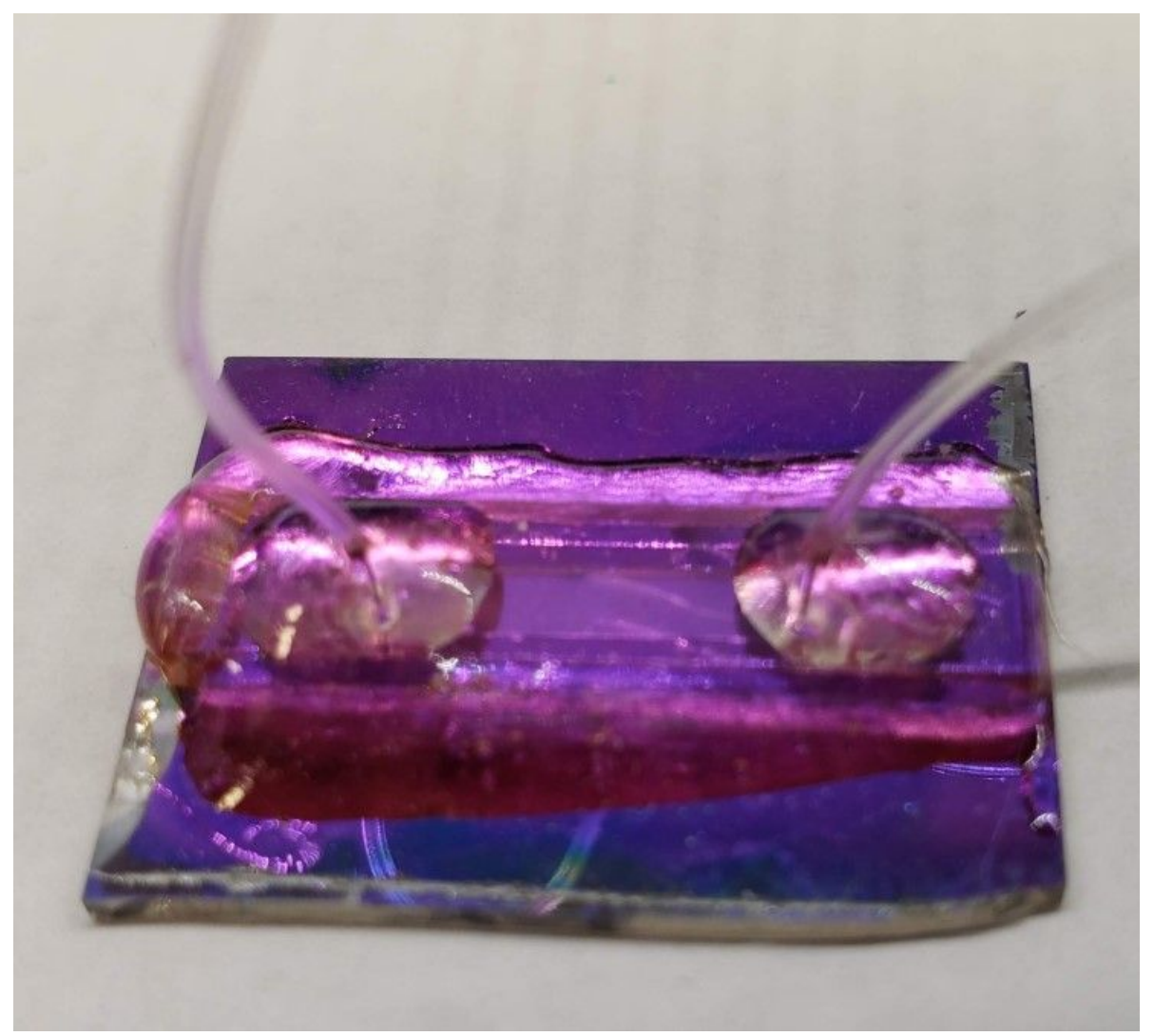

Figure S4: A photo of the fabricated thin-film based hydrogen gas sensor. 